\title{
Resultados de la Cirugía Laparoscópica en Quistes Hepáticos No Parasitarios
}

\author{
Results of Laparoscopic Surgery in Nonparasitic Hepatic Cysts
}

Carlos Manterola****

MANTEROLA, C. Resultados de la cirugía laparoscópica en quistes hepáticos no parasitarios. Int. J. Morphol., 31(3):1116-1119, 2013.

RESUMEN: Los quistes hepáticos no hidatídicos $(\mathrm{QHNH})$ se detectan de forma incidental, pueden ser únicos y múltiples. Desde que se describió el tratamiento laparoscópico de estos, se han reportado diversas series; existiendo por ende evidencia que avala la cirugía laparoscópica de estas lesiones. El objetivo de este estudio, es reportar los resultados del tratamiento laparoscópico de QHNH en términos de morbilidad postoperatoria (MPO). Serie de casos. Se incluyeron pacientes intervenidos quirúrgicamente vía laparoscópica por QHNH en el Hospital Clínico de la Universidad Mayor entre enero de 2008 y diciembre de 2012. El estudio preoperatorio consistió en exámenes generales, determinación de ELISA-IgE e IgG para hidatidosis, ecotomografía abdominal o tomografía computarizada. En el período estudiado, se intervinieron 17 pacientes con QHNH, resecándose en ellos, un total de 39 quistes. El 76,5\% de los casos eran de sexo femenino; y la mediana de edad de la serie fue de 56 años. La mediana del diámetro ecográfico de las lesiones fue de 7,5 cm. Se realizó quistectomía subtotal en todos ellos, sin utilización de drenaje. Se realizó epiploplastia en 4 pacientes. En el 82,4\% de los casos se ejecutó de forma simultánea una colecistectomía por colelitiasis. La mediana del tiempo quirúrgico fue de 51 minutos. No fue necesario convertir a ningún paciente. La serie no registra MPO ni mortalidad. La mediana de estancia hospitalaria fue de 1 día. El estudio histopatológico confirmó "quiste de tipo biliar" en la totalidad de los casos. Con un seguimiento mínimo de 6 meses, no se ha evidenciado MPO tardía ni reaparición de las lesiones resecadas. El tratamiento aplicado a esta serie de casos de QHNH, se asocia a escasa estancia hospitalaria y buena evolución a corto y mediano plazo.

PALABRAS CLAVE: Enfermedades hepáticas; Quiste simple del hígado; Quiste seroso del hígado; Quiste no parasitario del hígado; Enfermedad poliquística del hígado.

\section{INTRODUCCIÓN}

Los quistes hepáticos no hidatídicos (QHNH); pueden ser quistes simples o biliares y la enfermedad poliquística hepática, que suele asociarse a quistes simples en ambos riñones. Constituyen formaciones quísticas intrahepáticas, recubiertas por un epitelio simple cuyo contenido es un líquido claro de características acuosas. No se comunican con la vía biliar; y se los ha denominado quistes hepáticos benignos, quistes uniloculares, quistes solitarios del hígado, quistes no parasitarios o quiste hepáticos congénitos. Son siempre benignos y no se malignizan.

Si bien fueron considerados una entidad poco frecuente, se han reportado prevalencias en autopsias de adultos $1 \%$ al 5\% (Amendolara et al., 2012); siendo más frecuente en mujeres, y aunque en su inmensa mayoría son asíntomáticos, pueden hacerse sintomáticos en sujetos de 40 a 50 años (Hansman et al., 2001).
Con la aparición de la ultrasonografía y la tomografía su diagnóstico, a modo de hallazgo, se ha hecho más común; detectándose de forma incidental entre un $2,5 \%$ y $5 \%$ de la población estudiada (Karavias et al., 2000).

Habitualmente no requieren tratamiento, con excepción de aquellos múltiples, de gran tamaño, los que se complican, o que dificultan la realización de otros procedimientos quirúrgicos; subgrupos que se beneficiaría de la cirugía. Desde que se describió el tratamiento laparoscópico de estos en 1991, se ha reportado evidencia suficiente que avala el rol de la cirugía laparoscópica en estas lesiones.

El objetivo de este estudio, es reportar los resultados del tratamiento laparoscópico de QHNH en términos

\footnotetext{
* Departamento de Cirugía, Universidad de La Frontera, Temuco, Chile.

** Centro de Investigación en Ciencias Biomédicas, Universidad Autónoma de Chile, Temuco, Chile.

Parcialmente financiado por Proyecto DID-UFRO DI12-0037, Dirección de Investigación de la Universidad de La Frontera, Temuco, Chile.
} 
de morbilidad postoperatoria (MPO). El reporte de este estudio, se hizo de acuerdo a la lista de verificación para estudios observacionales descriptivos (Manterola \& Astudillo, 2013).

\section{MATERIAL Y MÉTODO}

Se diseñó un estudio de serie de casos. El estudio se realizó en el Hospital Clínico de la Universidad Mayor entre enero de 2008 y diciembre de 2012. Los participantes fueron sujetos portadores de lesiones quísticas hepáticas únicas o múltiples, sugerentes de QHNH. El estudio preoperatorio consistió en exámenes generales, pruebas de función hepática, ecotomografía abdominal o tomografía computarizada (Fig. 1); y determinación de ELISA IgE e IgG para hidatidosis. Se incluyeron pacientes intervenidos quirúrgicamente vía laparoscópica por QHNH. Se excluyeron pacientes con lesiones quísticas sugerentes de hidatidosis hepática.

Técnica. Con neumoperitoneo de $12 \mathrm{~mm} \mathrm{Hg}$, acceso americano con 4 trócares (dos de $10 \mathrm{~mm}$ y dos de $5 \mathrm{~mm}$ ); y luego de una exploración de la lesión (Fig. 2a), se procedió a puncionarlos y evacuarlos (Fig. 2b). Posteriormente, se efectuó una quistotomía amplia (Fig. 2c); y una exéresis del quiste hasta dejar tejido hepático cruento en el límite de sección (Fig. 2d). Sólo ocasionalmente se procedió a una epiploplastia.

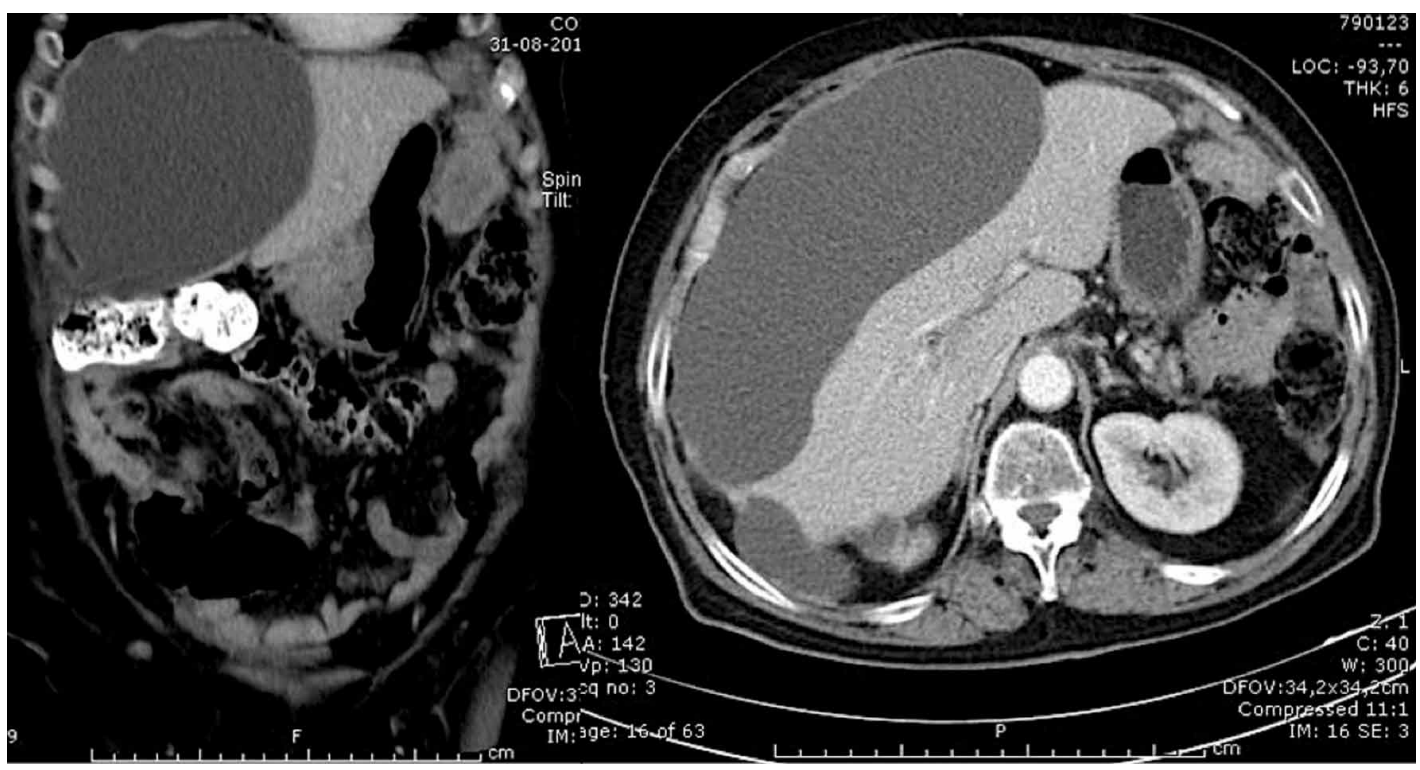

Fig. 1. Imágenes de tomografía axial computarizada de uno de los pacientes de la serie, que presentaba una extensa lesión quística única, que comprometía la totalidad del lóbulo derecho del hígado.

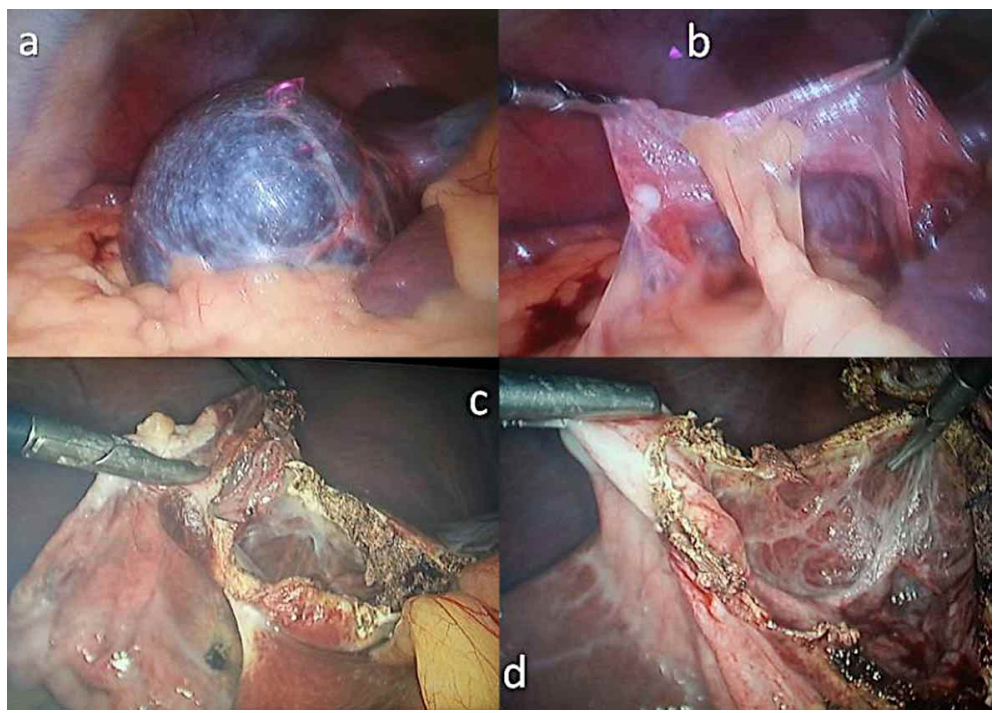

Fig. 2. Secuencia del procedimiento terapéutico laparoscópico. Se aprecia la lesión localizada en el segmento IV (2a); luego se observa esta una vez puncionada $(2 b)$; posteriormente una quistotomía extensa (2c); y finalmente una etapa avanzada de la resección del quiste, apreciándose el parénquima hepático cruento y el interior de la lesión (2d). 
Se utilizó un muestreo de tipo no probabilístico consecutivo. La variable resultado fue MPO, medida a 30 días del postoperatorio. Otras variables de interés fueron: tiempo quirúrgico, conversión, estancia hospitalaria, mortalidad y recidiva. En relación a su seguimiento, la totalidad de los especímenes resecados fueron a estudio histopatológico. Los pacientes fueron seguidos clínica y ecotomográficamente por un mínimo de 6 meses.

Se utilizó estadística descriptiva con cálculo de porcentajes, de medidas de tendencia central y dispersión. Todos los pacientes firmaron sus respectivos consentimientos informados y su identidad fue reservada utilizando códigos.

\section{RESULTADOS}

En el período estudiado, se intervinieron de este modo 17 pacientes con QHNH, resecándose un total de 39 quistes. El 76,5\% de los casos eran de sexo femenino; y la mediana de edad de la serie fue de 56 años.

La mediana del diámetro ecográfico de las lesiones fue de $7,5 \mathrm{~cm}$. Se realizó quistectomía subtotal en todos ellos, sin utilización de drenaje. Se realizó epiploplastia en 4 pacientes. Se trataba de sujetos con lesiones de diámetro superior a $10 \mathrm{~cm}$.

En el 82,4\% de los casos se realizó de forma simultánea una colecistectomía por colelitiasis. La mediana del tiempo quirúrgico fue 51 minutos. No fue necesario convertir a ningún paciente. La serie no registra MPO ni mortalidad.

La mediana de estancia hospitalaria fue de 1 día. El estudio histopatológico confirmó "quiste de tipo biliar" en la totalidad de los casos. Con un seguimiento mínimo de 6 meses, no se ha evidenciado morbilidad tardía ni recidiva de las lesiones resecadas.

\section{DISCUSIÓN}

Los QHNP surgen del epitelio de los conductos biliares y se encuentran tapizados por una capa de epitelio cuboideo, o con menor frecuencia escamoso columnar (Taylor \& Langer, 1998); son más frecuentes en la mujer, con una relación $4: 1 ;$ y no comprometen la función hepática (Caporale et al., 1993). Ocasionalmente pueden generar síntomas secundarios a complicaciones evolutivas de la (s) lesión (es); como ictericia obstructiva, perforación, hemorra- gia intraquística, rotura a peritoneo, torsión del pedículo, etc. (Fernández et al., 1986).

El tratamiento de estos pacientes es quirúrgico, siendo la mejor opción el acceso y tratamiento laparoscópico, cuyo primer reporte fue documentado en 1991 en Alemania (Z'graggen et al., 1991; Morino et al., 1994); a partir de cuándo la evidencia es hasta hoy suficientemente sólida para apoyar esta opción terapéutica.

Sin embargo, un hecho a considerar en el enfrentamiento de estas lesiones, es el riesgo de confundir un QHNP con un quiste hidatídico hepático, especialmente cuando se trata de un quiste solitario (Manterola et al., 2007).

Uno de los hechos de interés se refiere a la ausencia de morbilidad de esta serie, cuando esta variable se ha reportado hasta entre un 2\% y $7 \%$ de los casos (Mazza et al., 2009; Loehe et al., 2010). Otro, se refiere a la ausencia de conversión, lo que en otras series alcanza a más de un $7 \%$ (Treckmann et al., 2010); y otro punto de interés es la estancia hospitalaria, que se ha reportado entre tres y ocho días (Manterola et al.).

No obstante los buenos resultados observados, cabe señalar que se trata de una serie pequeña; a pesar que la gran mayoría de las previamente publicadas son de escaso número de sujetos operados (Fernández et al.; Zacherl et al., 2000; Palanivelu et al., 2007; Stambuk et al., 2008).

En conclusión se puede mencionar que el tratamiento aplicado a esta serie de casos de QHNH, se asoció a estancia hospitalaria reducida y buena evolución en el corto y mediano plazo.

MANTEROLA, C. Results of laparoscopic surgery in nonparasitic hepatic cysts. Int. J. Morphol., 31(3):1116-1119, 2013.

SUMMARY: Nonparasitic hepatic cysts (NPHC) can be detected incidentally, and may be single or multiple. Since described laparoscopic treatment of NPHC, several series have been reported, therefore there is enough evidence that support the role of laparoscopic surgery in the treatment of NPHC. The aim of this study is to report the results of laparoscopic treatment of QHNH in terms of postoperative morbidity (POM). Case series. Patients operated laparoscopically by NPHC in the Universidad Mayor Clinical Hospital, Temuco, between January 2008 and December 2012 were included. The preoperative evaluation consisted of general exams, ELISA-IgE and IgG determination for hydatid disease, abdominal ultrasonography or abdominal CT scan. During the study period, 17 patients with NPHC were operated, resecting them a total of 39 cysts. $76.5 \%$ of patients were female, and the median age of the series was 56 years. The median sonographic diameter of the lesions was $7.5 \mathrm{~cm}$. Subtotal cystectomy was performed in 
all of them, without using drainage. Epiploplasty was performed in 4 patients. In $82.4 \%$ of cases simultaneously cholecystectomy for cholelithiasis was performed. The median operative time was 51 minutes. Neither patient was converted. The series does not register MPO or mortality. The median hospital stay was 1 day. Histopathology confirmed the "biliary type cyst" in all cases. With a minimum follow up of 6 months, there is no evidence or late MPO or recurrence of the lesions resected. The treatment applied to this series of NPHC, is associated with short hospital stay and good outcome in the short and medium term.

KEY WORDS: Liver Diseases; Polycystic liver disease; Cysts/surgery; Nonparasitic hepatic cysts.

\section{REFERENCIAS BIBLIOGRÁFICAS}

Amendolara, M.; Bucca, D.; Barbarino, C.; Romano, M. F.; Marino, G.; Zucchelli, M.; Stevanato, G.; Bernardi, M. \& Ranzato, R. Surgical management of symptomatic simple hepatic cysts. G. Chir., 33(1-2):17-20, 2012.

Caporale, A.; Guilani, A.; Teneriello, F. L.; Cosenza, U. M. \& Costi, U. Surgical management of nonparasitic cysts of the liver: report 17 cases. Dig. Surg., 10(5):249-53, 1993.

Fernández, F. S. A.; Ortiz Aguilar, M.; Cortés Gómez, M.; Blond, A. B.; Tello López, J. I. \& Donis, S. J. Quiste hepático no parasitario. A propósito de cuatro observaciones. Rev. Esp. Enf. Apar. Digest., 70:79-84, 1986.

Hansman, M. F.; Ryan, J. A. Jr.; Holmes, J. H. 4th.; Hogan, S; Lee, F. T; Kramer, D. \& Biehl, T. Management and long-term follow up of hepatic cysts. Am. J. Surg., 181(5):404-10, 2001.

Karavias, D. D.; Tsamandas, A. C.; Payatakes, A. H.; Solomou, E.; Salakou, S.; Felekouras E. S. \& Tepekes, K. N. Simple (nonparasitic) liver cysts: clinical presentation and outcome. Hepatogastroenterology, 47(35):1439-43, 2000.

Loehe, F.; Globke, B.; Marnoto, R.; Bruns, C. J.; Graeb, C.; Winter, H.; Jauch, K. W. \& Angele, M. K. Long-term results after surgical treatment of nonparasitic hepatic cysts. Am. J. Surg., 200(1):23-31, 2010.

Manterola, C. \& Astudillo, P. Checklist for reporting of descriptive observational studies. MINCIR initiative. Int. J. Morphol., 31(1):115-20, 2013.

Manterola, C.; Pineda, V. \& Vial, M. Efectividad del tratamiento laparoscópico de quistes y tumores hepáticos. Revisión global de la evidencia. Rev. Chil. Cir., 59(4):264-71, 2007.

Mazza, O. M.; Fernandez, D. L.; Pekolj, J.; Pfaffen, G.; Sanchez Clariá, R.; Molmenti, E. P. \& de Santibañes, E. Management of nonparasitic hepatic cysts. J. Am. Coll. Surg., 209(6):7339, 2009.
Morino, M.; De Giuli, M.; Festa, V. \& Garrone, C. Laparoscopic management of symptomatic nonparasitic cysts of the liver. Indications and results. Ann. Surg., 219(2):157-64, 1994.

Palanivelu, C.; Rangarajan, M.; Senthilkumar, R. \& Madankumar, M. V. Laparoscopic Management of Symptomatic Multiple Hepatic Cysts: a Combination of Deroofing and Radical Excision. JSLS, 11(4):466-9, 2007.

Stambuk, J.; De Aretxabala, X.; Lavin, M. \& Zamarin, J. Fenestración laparoscópica de quistes hepáticos sintomáticos: Reporte de tres casos. Rev. Chil. Cir., 60(6):548-51, 2008.

Taylor, B. R. \& Langer, B. Current surgical management of hepatic cyst disease. Adv. Surg., 31:127-48, 1998.

Treckmann, J. W.; Paul, A.; Sgourakis, G.; Heuer, M.; Wandelt, M. $\&$ Sotiropoulos, G. C. Surgical treatment of nonparasitic cysts of the liver: Open versus laparoscopic treatment. Am. J. Surg., 199(6):776-81, 2010.

Zacherl, J.; Scheuba, C.; Imhof, M.; Jakesz, R. \& Függer, R. Longterm results after laparoscopic unroofing of solitary symptomatic congenital liver cysts. Surg. Endosc., 14(1):5962,2000

Z'graggen, K.; Metzgern, A. \& Kaiber, C. Syntomatic simple cysts of the liver: treatament by laparoscopic surgery. Surg. Endosc., 5(4):224-5, 1991.

\section{Dirección para Correspondencia: \\ Dr. Carlos Manterola \\ Departamento de Cirugía \\ Universidad de La Frontera. \\ Casilla 54-D, Temuco, Chile.}

Teléfono: $56-45-2325760$

Fax: 56-45-2325761

Email: carlos.manterola@ufrontera.cl

Recibido : 09-05-2013

Aceptado: 12-07-2013 